\title{
Die normative Praxis von Gesundheit und Krankheit
}

\begin{abstract}
The Normative Practice of Health and Disease. „Health“ and „disease“ are frequently used terms with a high relevance for our everyday lives. Their spectrum of meaning is very extensive, but also ambivalent, because they are not adequately captured by a purely medical-scientific approach. The forms of knowledge associated with „disease“ and „health“ are rather diverse and allow different ways of looking at them side by side in a justified manner. Against this background, the relationship between scientific and life world approaches to these phenomena is of central importance, because this results in very different claims to an interpretative sovereignty of "health“ and „disease“. For these states not merely have an associated dimension, but an essentially practical-normative one, so that they cannot simply be reduced to a biological function or dysfunction. This becomes especially clear when the assignment of dysfunctional conditions to the concept of disease results in immediate options for action that are not simply limited to a chapter expansion of medical textbooks, but may lead to fundamental personal and social changes. For this side of „disease“ and „health“ reflects not only medical developments, but also normative attitudes in science and society. These in turn are also decisive for the communicative structure in the doctorpatient relationship.
\end{abstract}

\section{Einführung: Gesundheit und Krankheit}

In unserer Lebenspraxis spielen die semantisch anspruchsvollen Begriffe „Gesundheit“ und „Krankheit“ eine wesentliche Rolle und sind oft in Gebrauch. Doch welcher Kompetenz es bedarf, über diese Begriffe, ihre Inhalte und ihre Praxisrelevanz aufzuklären, versteht sich nicht von selbst. Die Auskünfte, welche die Literatur, das Internet, Ärztinnen und Ärzte, Fitnessarmbänder, Selbsthilfegruppen oder auch das schlichte Hineinhorchen in uns selbst uns geben, werden sich nicht immer decken. $\mathrm{Zu}$ verschieden sind die Ansatzpunkte, die Erfahrungen und die Interessen der jeweiligen Protagonisten. Zudem ist unklar, nach welchem Typ von Wissen hier gefragt wird, wenn Krankheits- und Gesundheitszustände beschrieben werden sollen.

Dirk Lanzerath, Deutsches Referenzzentrum für Ethik in den Biowissenschaften (DRZE), Universität Bonn

Ә OpenAccess. (c) 2021 Dirk Lanzerath, publiziert von De Gruyter. (cc) BY-NC-SA Dieses Werk ist lizenziert unter einer Creative Commons Namensnennung - Nicht kommerziell - Weitergabe unter gleichen Bedingungen 4.0 International Lizenz. https://doi.org/10.1515/9783110713336-006 
Es fällt häufig leichter, „Krankheit“ - als den eher negativ konnotierten Begriff - zu beschreiben als „Gesundheit“. Der Zustand der Krankheit macht uns sehr unmittelbar unsere Natürlichkeit, Fragilität und Vergänglichkeit bewusst, während sich der Zustand der Gesundheit eher als eine verborgene Form von Anwesenheit erweist (vgl. Gadamer 1993, S. 133; Leder 1990, S. 79-83; 1995, S. 1007). Wir nehmen Gesundheit für gewöhnlich nicht wahr oder halten sie bestenfalls für selbstverständlich. Bewusst wird sie uns nur angesichts ihrer Negation. Erst durch den Zustand der Krankheit wird sie entborgen. Eine lebenspraktische und lebensweltliche Perspektive auf diese elementaren Grundbegriffe unseres Daseins scheint uns aber zunehmend auf eine eigenartige Weise verstellt. Denn in der Moderne ist nicht mehr primär dieser unmittelbare und ursprüngliche, lebensweltliche Blick auf die Zustände unseres Körpers dominant, vielmehr bestimmt ein neuer, datengesättigter, wesentlich naturwissenschaftlich geprägter und häufig als naturalistisch aufgefasster Blick einer naturwissenschaftlich arbeitenden Medizin unsere Vorstellungen darüber, was den Inhalt dieser Grundbegriffe ausmacht (Boorse 1981 [1975], 1997). Eine naturwissenschaftlich arbeitende Medizin scheint eine Art Definitionsmacht nicht nur darüber zu beanspruchen, was einzelne Krankheiten als körperliche oder mentale Dysfunktionalitäten ausmachen und wie ihre empirischen Parameter zu beschreiben sind, sondern auch über unsere anthropologischen Einordnungen von Krankheit und Gesundheit sowie die Bedeutung dieser Zustände für die gesellschaftlichen Zusammenhänge und für unsere individuellen Lebensentwürfe. Dabei hat der Arzt und Philosoph Karl Jaspers bereits Anfang des letzten Jahrhunderts bemerkt: „Was gesund und was krank im allgemeinen bedeute, darüber zerbricht sich der Mediziner am wenigsten den Kopf“ (Jaspers 1923, S. 4).

Die Bedeutungen von „Gesundheit“ und „Krankheit“ erweisen sich offensichtlich als wesentlich umfangreicher und werden durch einen rein medizinwissenschaftlichen Zugriff nicht hinreichend erfasst. Die Wissensformate, die sich mit „Krankheit“ und „Gesundheit“ verbinden, sind divers und lassen unterschiedliche Betrachtungsweisen zu.

\section{Wissenschaftliche Methoden und Lebenswelt}

Den Disziplinen der medizinischen Wissenschaft kommt sicherlich eine zentrale Bedeutung bei der Frage zu, was Krankheit und Gesundheit ausmacht. Methodisch betrachtet ist der Blick auf die Welt durch die wissenschaftliche Brille jedoch nur einer von vielen. Die vielfältigen Blicke durch lebensweltliche Brillen können hiervon erheblich abweichen und müssen nicht immer dem der Wissenschaften entsprechen. Dieser Topos vom Verhältnis zwischen Wissenschaft 
und Lebenswelt ist immer wieder thematisiert worden, so etwa besonders intensiv von Edmund Husserl. Er beschreibt in seiner Phänomenologie eine subjektbezogene Einheit von Ich und Welt, die sich durch eine intentionale Grundstruktur des Ich-erfahre-etwas auszeichnet. Hierin wird für ihn „Lebenswelt“ zu einem zentralen Begriff für einen vortheoretischen und noch unhinterfragten Zugang zur Welt. Die Lebenswelt ist die Welt der „natürlichen Einstellung“, die ,im schlichtesten Sinne bewusstseinsmäßig für uns da ist, die Welt, in der wir leben, denken, wirken, schaffen“ (Husserl 2002, S. 18).

In der Lebenswelt werden die Ich-Perspektive und der subjektive Blickwinkel nicht systematisch ausgeklammert, sondern sie sind geradezu der Bezugspunkt unserer vielfältigen Erfahrungs- und Erlebniswelten. Der wissenschaftliche Blick, der stets ein distanzierter und fragmentierter Blick auf die Welt sein muss, droht den Menschen von sich und der Welt zu entfremden, wenn Wissenschaften die alleinige Deutungshoheit über die Welt beanspruchen. Husserl versucht in seinem philosophischen Programm des frühen 20. Jahrhunderts (der transzendentalen Phänomenologie), die Entfremdungserscheinung zwischen Mensch und Welt, die sich gerade durch die Methoden der Naturwissenschaften sowie durch ihre wirtschaftlichen und technischen Anwendungen ausbreiten, wieder $\mathrm{zu}$ verringern. Denn die Wissenschaften befassen sich eben nicht mit der entscheidenden Frage, wer der Mensch denn ist und welchen Sinn seine Existenz hat (,für ein echtes Menschentum“) (Husserl 2002, § 2,4). Husserl bezeichnet dies als eine praktische Krise und als eine Krise des Wissenschaftsbegriffs, wie er sich herausgebildet hat mit der Entwicklung der naturwissenschaftlichen Disziplinen und wie Jürgen Mittelstraß es ausdrückt - der Entstehung der „Leonardo-Welten“, in denen Handwerkerstätten und wissenschaftliche Theorien sich erfolgreich miteinander verbinden (Mittelstraß 1992). Der naturwissenschaftlich-technische Fortschritt, den Francis Bacon im 17. Jahrhundert als Erleichterung für die Lebenspraxis vor Augen gehabt hat (Bacon 1990), entfaltet sich zum Ende des 19. Jahrhunderts zu einem Symbol lebensweltlicher Hoffnung. Im nachfolgenden Jahrhundert wird aber die Gesellschaft Zug um Zug mit den negativen Folgen dieser Praxis, wie Umweltzerstörung und Gesundheitsgefährdung, sowie schließlich im 21. Jahrhundert mit der genetisch-pharmakologischen Selbstmanipulation in immer stärkerem Ausmaß konfrontiert. Lebenswissen wird nur noch naturwissenschaftlich-verfügend und kaum noch im Sinne eines Orientierungswissens verstanden, denn - so Edmund Husserls Kritik - bloße „Tatsachenwissenschaften machen bloße Tatsachenmenschen“ (Husserl 2002, § 2,4). Husserls Intention liegt keineswegs darin, wissenschaftliches Denken und Arbeiten als schädlich aufzufassen, es gar zu negieren oder überhaupt wissenschaftsfeindlich zu operieren, vielmehr ist es seine Absicht, wissenschaftliches Wissen adäquat in die Lebenswelt zu integrieren und nicht wissenschaftliches Wissen selbst für die 
Lebenswelt zu halten. Da gerade die Naturwissenschaften und damit auch eine naturwissenschaftlich arbeitende Medizin notwendigerweise methodisch fragmentiert und reduktiv arbeiten, besteht daher stets die Gefahr, den Teil für das Ganze zu halten und dabei Organismus und Mensch zu übersehen. Nimmt man diese Zusammenhänge ernst, dann erwachsen aus den verschiedenen Perspektiven sehr unterschiedliche Deutungsansprüche, so auch in Bezug auf das, was „gesund“ und „krank“ für uns bedeuten.

\section{Deutungshoheit und Deutungspraxis}

$\mathrm{Zu}$ dieser Deutungsdimension gehört auch, dass man „Gesundheit“ und „Krankheit“ eine nicht nur beigeordnete, sondern eine wesentlich praktisch-normative Dimension einräumt und in ihnen nicht nur eine biologische Funktion bzw. Dysfunktion sieht. Dies wird vor allem dann deutlich, wenn sich aus der Zuordnung von dysfunktionalen Zuständen zum Krankheitsbegriff unmittelbare Handlungsoptionen ergeben, die sich nicht einfach auf eine Kapitelerweiterung medizinischer Lehrbücher beschränken, sondern zu persönlichen und gesellschaftlichen Veränderungen führen.

Denn diese Seite von „Krankheit“ und „Gesundheit“ reflektiert nicht nur medizinische Fortentwicklungen, sondern auch normative Einstellungen in Wissenschaft und Gesellschaft. So hat etwa die immer wieder modifizierte Einordnung des Zustands der Trauer im amerikanischen Klassifikationssystem des Diagnostischen und Statistischen Handbuchs Psychischer Störungen (DSM) stets für Diskussionen gesorgt, denn es versteht sich nicht von selbst, Trauer mit Krankheit in Verbindung zu bringen. Wenngleich sich eine langfristige Trauerreaktion durchaus in Form einer Depression manifestieren kann, ist die Trauer selbst für gewöhnlich nicht als Krankheit einzustufen. Sie kennzeichnet vielmehr eine gesunde und emotional reife Reaktion auf eine Verlusterfahrung ohne einen Krankheitswert, wenngleich Trauer mit einem Leidensdruck verbunden ist, der symptomatischen Krankheitszuständen gleichen kann. Im DSM-IV (1994/ rev. 2000) wird Trauer bereits der Kategorie „weitere klinisch relevante Probleme“ zugeordnet (V62.82). Wenn noch zwei Monate nach dem Verlust eines Menschen Symptome wie Schuldgefühle oder intensive Beschäftigung mit Gefühlen von Wertlosigkeit vorhanden sind oder auch der Betreffende in seinen Funktionen erheblich beeinträchtigt ist, gar halluzinatorische Erlebnisse hat, dann schlägt das DSM-IV die Diagnose der „Major Depression“ (296.2x) vor (Kersting et al. 2001). Doch nach der aktuellen Auflage, dem DSM-5 (2013), kann - bei hinzutreten einer Reihe von Symptomen wie etwa Antriebslosigkeit, Schlafstörungen, Appetitstörungen, Konzentrationsmangel oder Ängstlichkeit - bereits eine mehr 
als zwei Wochen andauernde Trauer als eine behandlungsbedürftige Krankheit dem Zustand der Depression zugeordnet werden und damit in einem sehr frühen Stadium zum Gegenstand therapeutischen Handelns werden. Wenngleich sich im DSM-5 darum bemüht wird, Trauer („grief“) nicht generell als Krankheit zu verstehen, sie von einer schweren depressiven Episode („major depressive episode $(\mathrm{MDE}) “) \mathrm{zu}$ trennen sowie beide Zustände als möglicherweise koexistent zu betrachten, suggeriert das Diagnose- und Klassifikationssystem DSM schon durch die Verkürzung der Zeiträume, dass Trauer die Konnotation von Krankheit oder Dysfunktion erhält und damit ein zentraler menschlicher Emotionsausdruck pathologisiert wird, wenn er zu lange andauert. Traditionell kennt etwa die katholische Kirche das Sechswochenamt und das Jahrgedächtnis, die Rechtsgeschichte kennt das Trauerjahr. Das sind Hinweise darauf, die Trauerzeit als ein anthropologisch wertvolles Moment zu betrachten. In der kritischen Diskussion zu dieser Einordnung wird keineswegs in Abrede gestellt, dass Trauer krank machen kann und Therapien ab einem bestimmten Stadium sinnvoll und notwendig sind. Aber es lässt sich in den modernen, funktional eingerichteten Gesellschaften eine Tendenz erkennen, negativ konnotierte Emotionen nicht mehr aushalten und rasch therapieren zu wollen.

Die Herausforderung, wissenschaftliches Wissen, wie das über Gesundheitsund Krankheitszustände funktional in unsere lebensweltlichen Ansprüche zu integrieren, bezieht sich nicht nur auf die Dynamik der Wissenschaften und ihrer teilweise geschlossenen Sprachspiele, sondern hängt auch mit der Schwierigkeit zusammen, lebensweltliche Probleme eindeutig der richtigen wissenschaftlichen Disziplin zuzuordnen. Hier sei etwa auf die aktuelle Diskussion in der Kinderund Jugendpsychiatrie bzw. -psychotherapie darüber verwiesen, in welchen Fällen Symptome einer Aufmerksamkeitsdefizit-Hyperaktivitätsstörung (ADHS) bei Kindern wirklich als Krankheit einzustufen sind, die Diagnose überhaupt im Einzelfall korrekt ist, das Phänomen psychopharmakologisch behandelbar ist oder ob es sich vielmehr um ein soziales Phänomen handelt, das auf Defizite im familiären Rahmen oder in den westlichen Erziehungs- oder Schulsystemen hinweist. Dass hier ein Problem vorliegt, wird von keiner Seite bestritten, aber wie es einzuschätzen und wie es anzugehen ist - ob biochemisch, systemisch, tiefenpsychologisch oder schulpolitisch -, ist nicht nur hochumstritten, sondern die Frage wird auch auf dem Rücken der Betroffenen ausgetragen. Dies gilt nicht zuletzt deshalb, weil die verschiedenen Ansätze für die lebensweltlich Beteiligten sehr unterschiedliche Konsequenzen haben, sowohl im Blick auf die Ursachenanalyse als auch hinsichtlich des Umgangs mit dem zur Disposition stehenden Zustand (Armstrong 2002; Lanzerath 2011, S. 257-264; Leuzinger-Bohleber et al. 2006; Walcher-Andris 2006). Hier zeigt sich sehr deutlich, dass sich die Frag- 
mentierung der Wissenschaften in Einzeldisziplinen nur schwer mit der geforderten lebensweltlichen Einheit verträgt.

Derartige Formen der zunehmenden Pathologisierung und Medikalisierung der Lebenswelt sind gekoppelt mit einseitig interpretierten und inflationär gebrauchten Krankheitsbegriffen. Daher ist es notwendig, den Krankheitsbegriff wieder in seiner Multidimensionalität zu betrachten und seine internen Konstitutionsverhältnisse zu klären.

\section{Die Macht der digitalen Verarbeitung und Verbreitung}

Die naturwissenschaftliche Perspektive auf Krankheit und Gesundheit ist nicht irgendeine, sondern eine zentrale, denn das Krankheitsgeschehen korrespondiert mit physiologischen Zuständen des menschlichen Körpers und dessen Interaktion mit seiner Umwelt. Wesentliche Aspekte dieser Zusammenhänge können mit naturwissenschaftlichen Methoden adäquat erfasst werden, wenngleich nicht vollständig. Denn methodisch sind die Naturwissenschaften und auch eine naturwissenschaftlich arbeitende Medizin beschränkt. In den modernen empirischen Wissenschaften werden Daten durch quantitative Analysen in definierten Bereichen generiert. Mit dieser selektiven Quantifizierung werden die Forschungsgegenstände fragmentiert. Damit schränkt sich der Blick auf den bearbeiteten Gegenstand genau auf diese Fragmente ein.

Quantifizierte Daten werden in den empirischen Wissenschaften digital aufbereitet, um sie operabel zu machen - und werden dabei scheinbar sprach- und kulturunabhängig in Erklärungs-Modelle umgewandelt. Hierüber entstehen Finanzmodelle, Organismusmodelle, Krankheitsmodelle usf. In ihnen werden Daten „praktisch“. Zunächst geschieht dies nur im Rahmen einer eingeschränkten wissenschaftsinternen Pragmatik, um mit ihnen weiterarbeiten zu können, aber schließlich beginnt man damit, die Daten und Modelle im nächsten Schritt in die Lebenswelt zu übertragen. Solche wissenschaftlichen Modelle erscheinen in diesem Prozess auf den ersten Blick wie objektivierte Abbilder der Lebenswirklichkeit, deren Wirklichkeitskompetenz ungeklärt ist (Bailer-Jones 2009; Cartwright 1999; Gedinat 2013) und eher in eine Diktatur der Einzelwissenschaften führt. Das semantische Dilemma dieser Modellübertragungen auf die Lebenswelt hat die Krise der COVID-19-Pandemie besonders deutlich gemacht mit der Frage, wie epidemiologische Modelle $\mathrm{zu}$ verantwortbaren politischen Entscheidungen führen sollen. Wissenschaftliche Modelle sind hier sehr begrenzt, denn das 
Übermaß an Sinnmöglichkeiten der Lebenswirklichkeit kann durch die Naturwissenschaften nicht eingeholt werden (Gabriel 2013, S. $127 \mathrm{f}$. und $248 \mathrm{ff}$.).

Wenn man zwischen Einzelwissenschaft und Lebenswelt keine hermeneutischen Zwischenschritte einbaut, dann droht sich die moderne Gesellschaft hinsichtlich der Weltdeutung und Weltaneignung zu entmachten; sie wird selbst zum hypothetischen Modell. Das von Algorithmen errechnete Modell wird zur wirkmächtigen maschinenarithmetischen Realität, die durch Verabredungen in einem wissenschaftlichen Weltbild („scientific image“) als gerechtfertigt erscheint. Die stops and goes werden durch Rechenmodelle gesetzt, die sich auch von den Entwicklern verselbständigen können und auf die der einzelne Mensch - wenn die Parameter erst einmal gesetzt sind - nur noch reagieren und in Bezug auf die er oft erst viel zu spät eingreifen kann. Modelle werden so komplex, dass nur noch ein Computer sie nachrechnen kann, und ihre Grundlage sind sehr begrenzte syntaktische und semantische Mittel (Sturma 2007, S. 140). Damit schiebt sich eine neu geschaffene und sich selbst weiterschaffende virtuelle Welt digitaler Daten und Modelle zwischen den erkennend-deutenden Menschen und die Wirklichkeit, so dass der zur Mathematisierung befähigte Mensch Gefahr läuft, nicht mehr Erschaffer von Hilfsmitteln für das Verständnis der Wirklichkeit zu sein - wofür sie ursprünglich gedacht waren -, sondern durch die Verselbständigung der Modelle zum bloßen Beobachter dieser neu geschaffenen Zwischenwelten zu werden. Fast unmerklich wirken sich diese Szenarien auf unser soziales Handeln und unsere sozialen Räume aus. Wir werden digital darüber informiert, was uns das Internet zum Kauf anrät, und unsere Karriere wird von virtuellen sozialen Netzen bestimmt. Was virtuelle Welten und Modelle mit unseren Daten machen, ist kaum mehr steuerbar. ${ }^{1}$ Wir geben damit zunehmend die Hermeneutik, die Interpretation der Daten aus der Hand.

Zunehmend entwickeln Technologiefirmen Gadgets, die diverse Daten über Körper und Verhalten abtasten, verarbeiten, umwandeln, interpretieren und an andere Dateninteressierte weiterleiten. Besonders verbreitet sind Fitness- oder auch Gesundheitsarmbänder. Frank Schirrmacher hat hierzu weitreichende Fragen aufgeworfen: „Werden solche Systeme eine neue Gesundheitsökonomie einleiten? Werden wir neue Metriken dafür entwickeln, bei wem sich Behandlung

1 So schreibt der Jurist und Informationstheoretiker Lawrence Lessig: „The code regulates. It implements values, or not. It enables freedoms, or disables them. It protects privacy, or promotes monitoring. People choose how the code does these things. People write the code. Thus the choice is not whether people will decide how cyberspace regulates. People - coders - will. The only choice is whether we collectively will have a role in their choice - and thus in determining how these values regulate - or whether collectively we will allow the coders to select our values for us“ (Lessig 2000; vgl. auch Lessig 2006). 
lohnt oder nicht? Gibt es individuelle Strafen für falsche Lebensführung?“ Sind die schönen Armbänder „nicht der Schlussstein der Quantifizierbarkeit des Einzelnen, der sich nun in nichts mehr vom Modell des „homo oeconomicus“ unterscheidet: eines Wesens, das ausschließlich einer Effizienz- und Kontrolllogik gehorcht?“ (Schirrmacher 2014). Die Debatte um eine COVID-19-Tracking-App in Folge der SARS-CoV2-Pandemie hat gezeigt, wie Gesellschaften auf solche Systeme reagieren. Die ganze Gesellschaft wird in ein großes soziales Experiment eingebunden, wie spezifische Gesundheitsdaten $\mathrm{zu}$ angeordneten Verhaltensnormen im Dienste der Gesundheit führen sollen (Klar \& Lanzerath 2020). Inwieweit es hierdurch zu Stigmatisierungs- und Diskriminierungswirkungen kommen wird, die möglicherweise auch noch auf falsch-positiven Alarmen fundieren, kann nur die Praxis klären (Leprince-Ringuet 2020). Wissenschaftler haben vor der Schaffung von Werkzeugen gewarnt, die eine Datenerfassung in großem Maßstab ermöglichen, da die Daten anfällig für Cyberattacken und/oder ernsthaften Missbrauch in Form einer beispiellosen Überwachung der gesamten Gesellschaft sind (Open Letter 2020). Über die Verwendung zur Eindämmung von COVID-19 durch „schleichende Überwachung“ hinaus könnten Regierungen die Krise ausnutzen, um Tracking-Daten von Bürgern zu erstellen und aufzubewahren, die theoretisch auch in anderen Kontexten wie der Strafverfolgung genutzt werden könnten (Klar 2020). Zudem wäre eine Verwendung der Daten denkbar, die es ermöglicht, den allgemeinen Gesundheitszustand der Bürger zu überwachen. Eine solche Utopie, wie sie etwa Juli Zeh (Zeh 2010) beschreibt, würde langfristig zu einer „Gesundheitsdiktatur“ führen, die genährt wird von einem rein szientifischen Blick auf Gesundheit und Krankheit.

Die Nutzung von Datenerhebungssystemen - ob bewusst oder unbewusst nimmt stetig zu. Freilich tragen sie Positives zur Gesundheit bei: Wenn das Smartphone meldet, dass man sich am Tag zu wenig bewegt hat, kann man diese Information positiv nutzen, um sich mehr $\mathrm{zu}$ bewegen und Herz-Kreislauferkrankungen vorzubeugen. Aber je nachdem, wie eine Technik aufgebaut ist, wird man sich nicht sicher sein können, wer außer mir selbst noch von meinen Daten erfährt oder erfahren kann ${ }^{2}$ und ob eine digitale Handlungsaufforderung mehr und mehr zur Handlungspflicht wird.

Der Mensch ist auf dem besten Wege - dem Ideal von Szientismus und szientifischem Naturalismus folgend -, die Hermeneutik seiner eigenen Lebenspraxis, eingebettet in einen Sinnhorizont eines Lebensplans, gegen die Automa-

2 Die auf diese Weise selbstregulierte Gesellschaft wirkt durch die Verschmelzung von Mensch und digitaler Abtastmaschine „normativ ohne langfristige Reflexion über Gesundheit, Effizienz oder Krankheit“ (Schirrmacher 2014). 
tismen algorithmischer Hermeneutik einzutauschen. Daraus kann sich der Mensch nicht durch noch bessere Rechenmodelle, sondern nur durch kreativen Widerspruch selbst befreien. Dieser Widerspruch kann im Zusammenhang mit Gesundheit und Krankheit gerade darin bestehen, die eigene Erlebniswelt und das interpretatorische Gespräch wieder mehr in den Mittelpunkt zu stellen und nicht nur als Beiwerk wissenschaftlicher Daten zu betrachten. Die Konstitutionsverhältnisse sind wieder zurechtzurücken.

In der hier vertretenen Einschätzung dessen, welche Rolle die Konzepte „Krankheit“ und „Gesundheit“ spielen, soll daher insbesondere vom Erleben des Kranken selbst und von der Arzt-Patienten-Beziehung ausgegangen werden. Daten (worunter u.a. Labordaten fallen) spielen hier durchaus eine wichtige Rolle, aber eben nicht die einzige und nicht diejenige, die die Krankheitswirklichkeit konstituiert.

\section{Erleben von Krankheit und Gesundheit: Elemente der Selbstauslegung}

Die Bewertung eines Zustands als Krankheit im Kontext der individuellen Lebensführung kann - je nach Schwere der Krankheit - den Betroffenen mit der Frage nach dem Sinn seines Daseins konfrontieren. Es ist eine Konfrontation mit der eigenen kontingenten Existenzweise, die im Negativum des Krankseins auch etwas Positives erkennen lässt.

Krankheit offenbart dem Menschen - vielleicht mehr als alle anderen Befindlichkeiten - die Gleichzeitigkeit von Identität und Nicht-Identität mit seinem Körper und Leib: Werde ich krank, so wird mir mein Körper fremd; er ist es, der mich krank macht, gleichzeitig bin ich es, der krank ist und der sich nicht vom kranken Körper distanzieren kann. ${ }^{3}$ Das Leibliche ist nichts Fertiges, es entsteht

3 Der Mensch ist sein Körper nur im Modus der Verkörperung, d.h. als Person in Form von Sprache, Religion, Lachen, Weinen u. a. (Plessner 1983, S.136 - 217). „Wenn wir das Verhältnis des Menschen zu seinem Leib und seine Verkörperung im sprachlichen, kulturellen und sozialen Handeln nur begreifen können, wenn wir ihn als Person interpretieren, nämlich als ein Ich, das eine unlösliche Einheit mit Selbst und Leib bildet, zugleich aber zu beiden in einem Verhältnis steht, dann wird erklärlich, warum das Wissen des Menschen um seine Grundvollzüge stets ausgesprochen oder unausgesprochen - vom Wissen um die Gegenmöglichkeit begleitet ist“ (Honnefelder 1994, S.104-134). Der Mensch, dem diese Verschränkungen der Modi bewusst sind, „,begreift das ihn umgebende Medium als ,Welt', nämlich als das Wirkliche, das vor dem leeren Hintergrund des Nichts steht. Zur Erkenntnis der Realität gehört deshalb auch die zumindest implizite Erkenntnis ihrer möglichen Nichtigkeit“ (Honnefelder 1994, S. 123). 
ständig neu, indem es sich verwirklicht, d.h. verkörpert. Verkörperung und Entkörperung werden in den Zuständen von gesund und krank sehr deutlich symbolisiert. Sich gesund erhalten kann als ein Modus der Verkörperung, krank werden als ein Modus der Entkörperung angesehen werden. Das Krankheitserleben führt uns die Unsicherheit dieser Welt, deren Teil wir sind, vor Augen (Heidegger 1977, § 47, § 51). Die Gesunden neigen dazu, die Welt der Kranken zu meiden, weil die Begegnung mit den Kranken sie an ihre eigene Kontingenz, Verwundbarkeit und Sterblichkeit erinnert.

Besonders ernste Krankheiten lassen vor diesem Hintergrund Fragen aufkommen, die unsere Lebensgewohnheiten in Frage stellen: „Warum ist das passiert?“, „Warum gerade jetzt?“, „Warum ausgerechnet mir?“ Die von uns gedachte Welt kann auf einmal in sich zusammenfallen: „Es ist alles sinnlos, ich habe etwas falsch gemacht und mein jetziger Zustand ist die Strafe hierfür." Historisch sind Krankheiten in verschiedenen Kulturen sogar als Strafe Gottes oder der Götter aufgefasst worden (Eckart 1998, S. 8-14). Krankheit kann aber auch in der modernen Gesellschaft für den Einzelnen zum Anlass für eine Gewissenserforschung und Ausgangspunkt für Sinnsuche und -findung werden. Das ganze Leben kann sich durch Krankheitserfahrung ändern und $\mathrm{zu}$ einer Änderung der Rangordnung der Lebensziele führen.

Als ein Zwischenergebnis kann festgehalten werden, dass weder naturalistische Zugänge zur Krankheit, die diese nur als biologische Dysfunktionen betrachten, noch konventionalistische Zugänge zu Krankheit und Gesundheit, die diese als primär gesellschaftliches Konstrukt betrachten, befriedigend Auskunft darüber geben können, was Krankheit und Gesundheit ausmacht. Es liegt näher, den Krankheitsbegriff als einen praktischen Begriff $\mathrm{zu}$ verstehen, also einen Handlungsbegriff, der mit dem Erleben der kranken Person beginnt und im ArztPatient-Verhältnis über die Interpretation medizinischer Daten verfeinert wird. Um diese Interpretation kann dann auch gerungen werden (Lanzerath 2000, 2007, 2008). Hat man sich geeinigt, kann die Krankheitsfeststellung eine ganze Handlungskaskade auslösen und rechtfertigen.

\section{Kranker und Arzt}

Die Kommunikation zwischen Arzt und Patient sollte es dem Patienten ermöglichen, seinen Krankheitszustand besser zu verstehen. Der Patient verlangt damit aber in erster Linie nicht nach einer wissenschaftlichen Erklärung physischer Symptome, sondern er möchte die persönliche Situation verstehen, die sein Kranksein ausmacht. Der Kommunikationsprozess drückt die Bedeutung der Krankheit im Kontext der spezifischen biographischen Situation des Patienten 
aus (Toombs 1992, S. 110 -111; Volkenandt 2015). Die praktische Aufgabe besteht dann in einer Kontextualisierung des Erlebnisprozesses.

Auch hier stellt sich die Digitalisierung als eine große Herausforderung für beide Seiten dar. Der moderne Mensch hat sich oft vor dem Arztbesuch schon im Internet über seine Symptome und mögliche Therapien informiert. Dies einzuordnen und ernst zu nehmen, ist Aufgabe des Arztes. Dass das Internet den Arzt nicht ersetzt, sollte zur Patienteneinsicht gehören.

Für eine funktionierende Kommunikation zwischen Arzt und Patient sind die Tugenden des Patienten ebenso einzufordern wie die des Arztes, denn ohne den Willen zur Genesung nützen ärztliche Anstrengungen wenig (May 1956, S. 1112). So entwickelt sich in der kommunikativen Praxis zwischen Patient und Arzt ein praktischer Krankheitsbegriff, also ein Handlungsbegriff, der auf beiden Seiten mit bestimmten Erwartungen verbunden ist. Im Dialog zeigen sich gegenseitige Verantwortlichkeit und Solidarität. Das Handeln mit dem Anderen und an dem Anderen beginnt dann nicht erst mit dem physischen Akt, sondern bereits mit der Sprache, mit der Art der Kommunikation, die in der Medizin häufig völlig unterbewertet wird, auch in der monetären Honorierung (Volkenandt 2015). Dazu gehören nicht nur der vertrauliche Umgang mit allen erhobenen Daten und die Schweigepflicht des Arztes, sondern auch ein grundsätzliches Vertrauensverhältnis, das aber nur dann vorausgesetzt werden kann, wenn ärztliches Handeln sich an wohldefinierten Zielen orientiert.

Gerade hier lässt sich ein steter Wandel in Gesellschaft und Medizin erkennen, der neue medizinische Normen setzt und andere Gesundheitswirklichkeiten generiert.

\section{Die alten und neuen Normen der Medizin: Enhancement und Ökonomisierung}

Traditionell orientiert sich die Medizin an den beschriebenen praktischen Krankheits- und Gesundheitsbegriffen. Doch derzeit macht die Medizin in der modernen Gesellschaft einen fundamentalen Strukturwandel durch, der erhebliche Konsequenzen auch für unser Verständnis von Krankheit und Gesundheit hat.

Diese traditionellen Ziele (Heilung, Prävention, Linderung) ärztlichen Handelns werden vor dem Hintergrund der erweiterten Handlungsmöglichkeiten der modernen Medizin durch neue Ziele ergänzt oder ersetzt. Zunehmend wird die „medizintechnische Machbarkeit“ in den Mittelpunkt von Überlegungen und Handlungen innerhalb der Medizin gestellt, die diese zur „Anthropotechnik“ werden lassen. Das Bestreben der Menschen, ihre Leistungen immer weiter stei- 
gern zu wollen, alte Grenzen zu überschreiten und in diesen Prozess auch die eigene Gesundheit mit einzubeziehen, führt seit der Aufklärung zu jener Vorstellung, die davon ausgeht, dass für das menschliche Handeln potenziell keine Grenzen mehr bestehen. Kontingenz und Sterblichkeit, als Kennzeichen für die Natürlichkeit des Menschen, werden letztlich verdrängt. Der Glaube vieler Menschen an die medizintechnische Machbarkeit kann schließlich zu jener völligen Medikalisierung (Lanzerath 2002, 2021 (im Druck)) der Lebenswelt führen, die suggeriert, auch die sozialen Probleme seien letztlich medizinisch lösbar (vgl. WHO-Gesundheitsdefinition 1946) (World Health Organization 1948). Damit werden Intension und Extension von Krankheit und Gesundheit erheblich erweitert.

Der naturwissenschaftliche Einfluss auf die Medizin - der in seiner positiven Wirkung keineswegs in Frage gestellt werden soll, aber zu der Annahme verleitet, Medizin sei lediglich eine angewandte (Natur-)Wissenschaft - löst die traditionelle ärztliche Teleologie, also die Bindung an ein klares Ethos (Lanzerath 2015) auf und verwandelt die Medizin von einer techné in eine Technik. Eine solche Technik kann dann prinzipiell jedermann zu beliebigen Zwecken zur Verfügung stehen. Als Ziele einer so konstituierten modernen „Dienstleistungsmedizin“ werden u.a. die Verbesserung der Lebensqualität sowie das Erreichen eines „perfekten“ Gesundheitszustands diskutiert. Der Wunsch nach „Steigerung“ und „Verbesserung“ („Enhancement“) der menschlichen Natur wird beflügelt durch die Utopie einer leidensfreien Gesellschaft (vgl. Abschnitt 2).

Angeregt durch Bio- und Medizintechniken sowie verführt durch Utopien menschlicher Vervollkommnung, erstreben wir eine Art „zweite Gesundheit“, indem wir unsere natürliche Natur nicht mehr nur zu gestalten, sondern zu überwinden trachten. Das wachsende Wissen um die physischen und psychischen Zustände des Menschen suggeriert, man könne zur Steigerung der Lebensqualität mit Hilfe von konventionellen chirurgischen Eingriffen, genetischen Manipulationen oder Medikamenten langfristig den perfekten Menschen schaffen. Nicht nur Bereiche der kosmetischen Chirurgie oder die Einnahme von Antidepressiva als Lifestyle-Medikamenten, sondern auch die Verabreichung von Anabolika oder Amphetaminen durch die Sportmedizin zur Leistungssteigerung von Sportlern fügen sich in die Vorstellungen ein, dass das medizinische Telos sich nicht mehr in der Erhaltung der Gesundheit erschöpft. Wird etwa Doping in der Öffentlichkeit primär unter dem Aspekt der Wettbewerbsverzerrung kritisch diskutiert, so sind es darüber hinaus die Zielsetzung ärztlichen Handelns und das ärztliche Ethos, die hier zur Disposition stehen (Murray 1984; ZEKO 2009).

Durch die Ökonomisierung der Medizin und die Schwierigkeiten bei der Allokation von knappen Ressourcen im Gesundheitswesen entwickeln sich „Zubrote“ sowohl bei niedergelassenen Ärzten als auch bei Kliniken. Die so ge- 
nannten individuellen Gesundheitsleistungen (IGeL) sollen die Praxis, die ästhetische Chirurgie, die Klinik betriebswirtschaftlich überleben lassen. Je breiter eine gesellschaftliche Zustimmung für nicht mehr unmittelbar gesundheitsrelevante Leistungen wird, desto weniger Möglichkeiten berufsethisch motivierter Sanktionen wird es geben. Die Tendenzen der Ökonomisierung der Medizin und die gesteigerten Ansprüche in der Gesellschaft scheinen eine zunehmende Integration von Enhancement und Anthropotechniken in das ärztliche Handeln zu fördern. Es wird dann immer schwieriger, zu beurteilen, welche Rechtfertigung für eine ärztliche Leistung tatsächlich vorliegt (ZEKO 2013).

Angesichts der immer zahlreicher werdenden medizintechnischen Serviceleistungen vollzieht sich auf diese Weise ein Wandel vom „Patienten“ zum „Kunden“. Ein solches „ärztliches“ Handeln könnte schließlich marktgerecht über Angebot und Nachfrage geregelt und das bislang an die ärztliche Teleologie gebundene Arzt-Patient-Vertrauensverhältnis durch ein reines, individuelles Vertragsverhältnis ersetzt werden. Es bleibt zu fragen, ob die Gesellschaft ein Interesse an einer derartigen Rolle des Arztes als Dienstleister hat und wie sich diese Rolle zur Garantenstellung des Arztes verhält.

Freilich ist die Medizin als Teil einer Gesellschaft sozialethischen Werten, politischen und ökonomischen Kalkülen ausgesetzt. Mannigfache Geschmäcke, Wünsche und Phantasien beeinflussen die Ziel- und Prioritätensetzung in der Medizin. Gesellschaftliche Ziele und medizinische Ziele verweisen aufeinander und müssen gleichermaßen reflektiert werden. Die Frage, ob es überhaupt medizinische Zielsetzungen mit einem universellen oder globalen, d.h. transkulturellen Anspruch gibt, hängt sehr von der Auffassung ab, die man vom ärztlichen Handeln und von den in der Natur des Menschen angelegten universellen Erfahrungen von Krankheit hat. Aber erst dann, wenn sich die Begriffe „Krankheit“ und „Gesundheit“ klarer eingrenzen lassen, ist es möglich, diese Begriffe auch reflektiert in ethischen Zusammenhängen anzuwenden. Dies gilt besonders dann, wenn die traditionelle Struktur ärztlichen Handelns mehr und mehr in Frage gestellt wird.

\section{Fazit}

Gegenüber anderen Begriffen - die auch normativen Charakter haben können, aber extensional sehr offen sind - hat der praktische Krankheitsbegriff den Vorteil, Medizin und ärztliches Handeln eng zu fassen und berechenbar zu gestalten. Der so entwickelte Krankheitsbegriff orientiert sich daran, Kranksein als eine Weise des Mensch-Seins so zu fassen, dass die kommunikative Komponente des seine Befindlichkeit mitteilenden Menschen wesentlich zur Konstitution von 
Krankheit gehört. Dabei erweist sich der Arzt als jene Instanz, die dem um Selbstauslegung bemühten Kranken nicht nur im engeren Sinne therapeutische, sondern auch - gegen technizistische Verkürzungen - hermeneutische Hilfestellung gibt; der Arzt hilft dem Kranken, sein Krankheitserleben besser verstehen zu können (Lanzerath 2000).

Will man diesen hohen Anspruch einlösen, dann muss dem Arzt-PatientVerhältnis wieder ein breiterer Raum eingeräumt werden. Diese Funktion macht den Krankheitsbegriff zu einer Größe, die in einer durch nichts zu ersetzenden Weise das ärztliche Handeln spezifiziert, legitimiert und limitiert. Ein solcher praktischer Krankheitsbegriff kann dazu beitragen, dass Medizin weiterhin berechenbar bleibt, das Vertrauen zwischen Arzt und Patient stabilisiert wird und die innovativen Möglichkeiten moderner biomedizinischer Forschung im Rahmen von Diagnose und Therapie genutzt werden können, ohne dass die mit ihnen verbundenen Risiken eskalieren, die Medizin zur „Anthropotechnik“ wird (vgl. ausführlicher Fuchs et al. 2002) und die Medizinethik nur noch zur Technikfolgenabschätzung degradiert würde. Hierzu ist es erforderlich, die Daten der medizinischen Disziplinen sorgfältig in einen größeren Kontext der Erlebniswelt der Patientinnen und Patienten $\mathrm{zu}$ integrieren und nicht die Daten selbst für die Erklärung der Phänomene zu halten.

\section{Literatur}

Armstrong, Thomas (2002): Das Märchen vom ADHS-Kind: 50 sanfte Möglichkeiten, das Verhalten ihres Kindes ohne Zwang und ohne Pharmaka zu verbessern. Paderborn: Junfermann.

Bacon, Francis (1990): Neues Organon. Hamburg: Meiner.

Bailer-Jones, Daniela (2009): Scientific Models in Philosophy of Science. Pittsburgh: University of Pittsburgh Press.

Boorse, Christopher (1981 [1975]): „On the Distinction between Disease and Illness“. In: Arthur L. Caplan/H. Tristram Engelhardt Jr./James J. McCartney (Hrsg.): Concepts of Health and Disease. Interdisciplinary Perspectives. Reading, MA: Addison-Wesley, S. 545-560.

Boorse, Christopher (1997): „A Rebuttal on Health“. In: James M. Humber/Robert F. Almeder (Hrsg.): What is a Disease? Totowa, NJ: Humana Press, S. 1-134.

Cartwright, Nancy (1999): The Dappled World. A Study of the Boundaries of Science. Cambridge: Cambridge University Press.

Eckart, Wolfgang U. (1998): Geschichte der Medizin (3. Aufl.). Berlin: Springer.

Fuchs, Michael/Lanzerath, Dirk/Hillebrand, Ingo et al. (2002): Enhancement: Die ethische Diskussion über biomedizinische Verbesserungen des Menschen. Bonn: DRZE.

Gabriel, Markus (2013): Warum es die Welt nicht gibt. Berlin: Ullstein.

Gadamer, Hans-Georg (1993): Über die Verborgenheit der Gesundheit. Aufsätze und Vorträge. Frankfurt am Main: Suhrkamp. 
Gedinat, Jürgen (2013): Ein Modell von Welt. Unterwegs in der Globalisierung. Freiburg: Centaurus.

Heidegger, Martin (1977): Gesamtausgabe. Ausgabe letzter Hand. Bd. 2: Sein und Zeit. Frankfurt am Main: Vittorio Klostermann.

Honnefelder, Ludger (1994): „Das Verhältnis des Menschen zu Leben, Leiblichkeit, Krankheit und Tod. Elemente einer philosophischen Anthropologie“. In: Ludger Honnefelder/Günther Rager (Hrsg.): Ärztliches Urteilen und Handeln. Zur Grundlegung einer medizinischen Ethik. Frankfurt am Main: Insel, S. 104-134.

Husserl, Edmund (2002): Natur und Geist: Vorlesungen Sommersemester 1919. Dordrecht: Springer Netherlands.

Jaspers, Karl (1923): Allgemeine Psychopathologie für Studierende, Ärzte und Psychologen (3., vermehrte u. verbesserte Aufl.). Berlin \& Heidelberg: Springer.

Kersting, Anette/Reutemann, Michael/Ohrmann, Patricia et al. (2001): „Traumatische Trauer ein eigenständiges Krankheitsbild?“. In: Psychotherapeut 46(5), S. 301-308.

Klar, Renate (2020): „Ethical Dimensions of COVID-19 Tracking Apps: Privacy-Preserving Proximity Tracing Pepp-Pt and Data Protection“. SHERPA Blog. https://www.projectsherpa.eu/ethical-dimensions-of-covid-19-tracking-apps-privacy-preserving-proximitytracing-pepp-pt-and-data-protection//, besucht am 15.6.2020.

Klar, Renate/Lanzerath, Dirk (2020): „The Ethics of COVID-19 Tracking Apps -Challenges and Voluntariness“. In: Research Ethics 16(3-4), S. 1-9.

Lanzerath, Dirk (2000): Krankheit und ärztliches Handeln. Zur Funktion des Krankheitsbegriffs in der medizinischen Ethik. Freiburg: Karl Alber.

Lanzerath, Dirk (2002): „Enhancement: Form der Vervollkommnung des Menschen durch Medikalisierung der Lebenswelt?“. In: Ludger Honnefelder/Christian Streffer (Hrsg.): Jahrbuch für Wissenschaft und Ethik, Bd. 7. Berlin: De Gruyter, S. 319-336.

Lanzerath, Dirk (2007): „Der Begriff der Krankheit. Biologische Dysfunktion und menschliche Natur“. In: Ludger Honnefelder/Matthias C. Schmidt (Hrsg.): Naturalismus als Paradigma. Wie weit reicht die naturwissenschaftliche Erklärung des Menschen? Berlin: Berlin University Press, S. 215-235.

Lanzerath, Dirk (2008): „Was ist medizinische Indikation? Eine medizinethische Überlegung“. In: Ralf Charbonnier/Klaus Dörner/Steffen Simon (Hrsg.): Medizinische Indikation und Patientenwille. Behandlungsentscheidungen in der Intensivmedizin und am Lebensende. Stuttgart: Schattauer, S. 35-52.

Lanzerath, Dirk (2011): „Professionsethische Aspekte aktueller Praktiken der Optimierung der menschlichen Natur“. In: Willy Viehöver/Peter Wehling (Hrsg.): Entgrenzung der Medizin. Von der Heilkunst zur Verbesserung des Menschen? Bielefeld: Transcript, S. 251-270.

Lanzerath, Dirk (2015): „Ethos“. In: Dieter Sturma/Bert Heinrichs (Hrsg.): Handbuch der Bioethik. Stuttgart: J. B. Metzler, S. 35-43.

Lanzerath, Dirk (2021 (im Druck)): „Medikalisierung“. In: Michael Fuchs (Hrsg.): Handbuch Alter und Altern. Anthropologie - Kultur - Ethik. Stuttgart: J. B. Metzler.

Leder, Drew (1990): The Absent Body. Chicago: University of Chicago Press.

Leder, Drew (1995): „Health and Disease. The Experience of Health and Illness“. In: Warren T. Reich (Hrsg.): Encyclopedia of Bioethics, Bd. 3. New York, NY: Macmillan, S. 1106-1113. Leprince-Ringuet, Daphne (2020): „Contact-tracing Apps: Why the NHS Said No to Apple and Google's Plan“. https://www.zdnet.com/article/contact-tracing-apps-why-the-nhs-said-noto-apple-and-googles-plan/, besucht am 30.5.2020. 
Lessig, Lawrence (2000): „Code is Law. On Liberty in Cyberspace“. Harvard Magazine. https:// harvardmagazine.com/2000/01/code-is-law-html, besucht am 12.1.2020.

Lessig, Lawrence (2006): Code: Version 2.0. New York, NY: Basic Books.

Leuzinger-Bohleber, Marianne/Brandl, Yvonne/Hüther, Gerald (2006): ADHS - Frühprävention statt Medikalisierung. Theorie, Forschung, Kontroversen. Göttingen: Vandenhoeck \& Ruprecht.

May, Eduard (1956): Heilen und Denken. Berlin: Dr. Georg Lüttke.

Mittelstraß, Jürgen (1992): Leonardo-Welt: Über Wissenschaft, Forschung und Verantwortung. Frankfurt am Main: Suhrkamp.

Murray, Thomas H. (1984): „Drugs, Sports, and Ethics“. In: Thomas H. Murray/Willard Gaylin/Ruth Macklin (Hrsg.): Feeling Good and Doing Better. Ethics and Nontherapeutic Drug Use. Clifton, NJ: Humana Press, S. 107-129.

Open Letter (2020): „Joint Statement on Contact Tracing: Date 19th April 2020“. https://drive. google.com/file/d/10Qg2dxPu-x-RZzETlpV3IFa259Nrpk1 J/view, besucht am 20.6.2020.

Plessner, Helmuth (1983): „Die Frage nach der Conditio humana“. In: Helmuth Plessner (Hrsg.): Gesammelte Schriften, Bd. 8. Frankfurt am Main: Suhrkamp, S. 136-217.

Schirrmacher, Frank (2014): „Das Armband der Neelie Kroe“. FAZ 51, S. 9.

Sturma, Dieter (2007): „Freiheit im Raum der Gründe. Praktische Selbstverhältnisse und die neurophilosophische Herausforderung“. In: Ludger Honnefelder/Matthias C. Schmidt (Hrsg.): Naturalismus als Paradigma. Wie weit reicht die naturwissenschaftliche Erklärung des Menschen? Berlin: Berlin University Press, S. 138-153.

Toombs, S. Kay (1992): The Meaning of Illness. A Phenomenological Account of the Different Perspectives of Physician and Patient. Dordrecht: Springer Netherlands.

Volkenandt, Matthias (2015): „Patient-Arzt-Kommunikation: Aufforderung zum ,empathischen Tanz““. In: Rheinisches Ärzteblatt 11, S. 28-29.

Walcher-Andris, Elfriede (2006): „Ethische Aspekte des pharmakologischen „cognition enhancement" am Beispiel des Gebrauchs von Psychostimulanzien durch Kinder und Jugendliche“. In: Ethik in der Medizin 18(1), S. 27-36.

World Health Organization (1948): „Preamble to the Constitution of WHO as adopted by the International Health Conference, New York, 19 June-22 July 1946; signed on 22 July 1946 by the representatives of 61 States (Official Records of WHO, no. 2, p. 100) and entered into force on 7 April 1948. The definition has not been amended since 1948“. https:// www.who.int/about/who-we-are/frequently-asked-questions, besucht am 13.7.2020.

Zeh, Juli (2010): Das Mögliche und die Möglichkeiten: Rede an die Abiturienten des Jahrgangs 2010. Saarbrücken: Gollenstein.

ZEKO (2009): „Bekanntmachungen: Stellungnahme der Zentralen Kommission zur Wahrung ethischer Grundsätze in der Medizin und ihren Grenzgebieten (Zentrale Ethikkommission) bei der Bundesärztekammer zur Doping und ärztliche Ethik“. In: Deutsches Ärzteblatt International 106(8), S. A-360-364.

ZEKO (2013): „Stellungnahme der Zentralen Kommission zur Wahrung ethischer Grundsätze in der Medizin und ihren Grenzgebieten (Zentrale Ethikkommission) bei der Bundesärztekammer: ,Ärztliches Handeln zwischen Berufsethos und Ökonomisierung. Das Beispiel der Verträge mit leitenden Klinikärztinnen und -ärzten““. In: Deutsches Ärzteblatt International 110(38), S. A-1752-1756. 